\title{
CONTROL OF VIBRATIONAL FIELD IN AN ELASTIC VIBRATION UNIT WITH DC MOTORS AND TIME-VARYING OBSERVER
}

\author{
Olga P. Tomchina \\ Saint Petersburg State University of Architecture and Civil Engineering (SPSUACE), \\ 2-nd Krasnoarmeiskaya St. 4, 190005, St. Petersburg, Russian Federation \\ otomchina@mail.ru
}

Article history:

Received 12.12.2021, Accepted 27.12.2021

\begin{abstract}
In the paper the problem of feedback control of vibrational fields in a vibration unit is analyzed taking into account the influence of the elasticity of cardan shafts, the drive dynamics, saturation for control torques. In addition, the synthesized rotor synchronization control algorithm uses the estimates of a non-stationary observer, which makes it possible to implement it practically on a two-rotor vibration unit SV-2. The performance of the closed loop mechatronic systems is examined by simulation for the model of the two-rotor vibration unit SV-2.
\end{abstract}

\section{Introduction}

The use of computer technologies for control of technical systems allows for the implementation of more complex control algorithms that ensure high quality processes. One of the main conditions in the design of control systems for mechatronic vibration units (VU), intended for screening bulk materials, is to ensure an acceptable shape and vibration parameters that ensure such a course of the process in which tangible technological advantages can be obtained. A feature of two-rotor vibrating screens is the complex shape of vibrations of the working body (platform): different points of the working body of these machines move along different trajectories, which creates opportunities for optimizing work processes .

Recently, there has been an increased interest in the development of control algorithms for VU, in which vibration fields corresponding to inhomogeneous vibration fields of a given type are realized [Gouskov et al., 2021; Chen et al., 2021; Tomchina, 2018]. The field characteristics are understood as the semi-axes of elliptical trajectories at arbitrary points of the platform and the angles of inclination of these axes. There are indications that the use of such fields has a number of technolog- ical advantages [el.al., 2001; Firsova, 2002]. In these works, the type of the vibration field was determined based on the model of the dynamics of the mechanical part of the VU. It is shown in [el.al., 2001; Firsova, 2002] that in the synchronization mode with constant mass-inertial parameters of vibration exciters of a tworotor unit and with fixed coordinates of their attachment points on the platform, the type of vibration field is determined by the phase shift of the unbalanced rotors. In mechatronic VUs, a stable synchronization mode with a constant specified phase shift can be realized through the use of special control algorithms.

The control algorithms for the synchronization of the rotating rotors of a two-rotor vibratory unit can be effectively synthesized on the basis of the speed gradient method [Fradkov, 1980; Andrievsky and Fradkov, 2021], which allows one to design control algorithms for substantially nonlinear systems. In this case, the goal functional $Q(p, q)$ is selected on the basis of the total energy of the mechanical system without taking into account friction losses, since in this case the total energy is invariant for the mechanical system, which is what the speed gradient method requires. However, the technical implementation of such algorithms requires information on the full state vector of the mechanical subsystem, which is not always feasible. This problem can be solved by using a non-stationary (time-varying) observer [Tomchina, 2020].

The issues of studying the type of vibration fields for models of two-rotor mechatronic vibration units, which took into account not only the dynamics of the mechanical part of the VU, but also the dynamics of the electric drive system were considered in [Fradkov et al., 2021; Tomchina, 2019; Tomchina et al., 2019].

However, an important factor in the analysis of the effectiveness of control algorithms for electromechanical systems of a VU is to take into account in the model of 


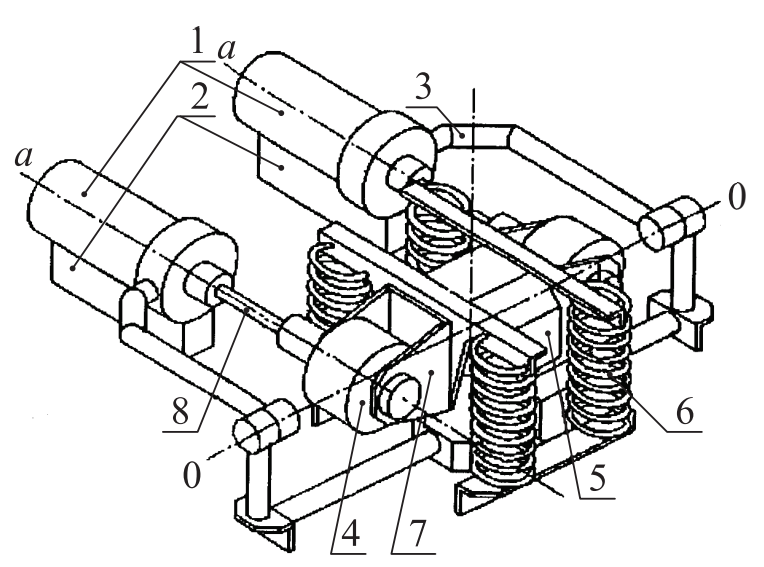

Figure 2. Schematics of two-rotor vibration unit: 1 - motors; $2-$ motor supports; 3 - frame of the unit; 4 - unbalanced rotors; 6 - vibrating platform; 7 - springs, 8 - rotor bearings; 9 - cardan shafts.

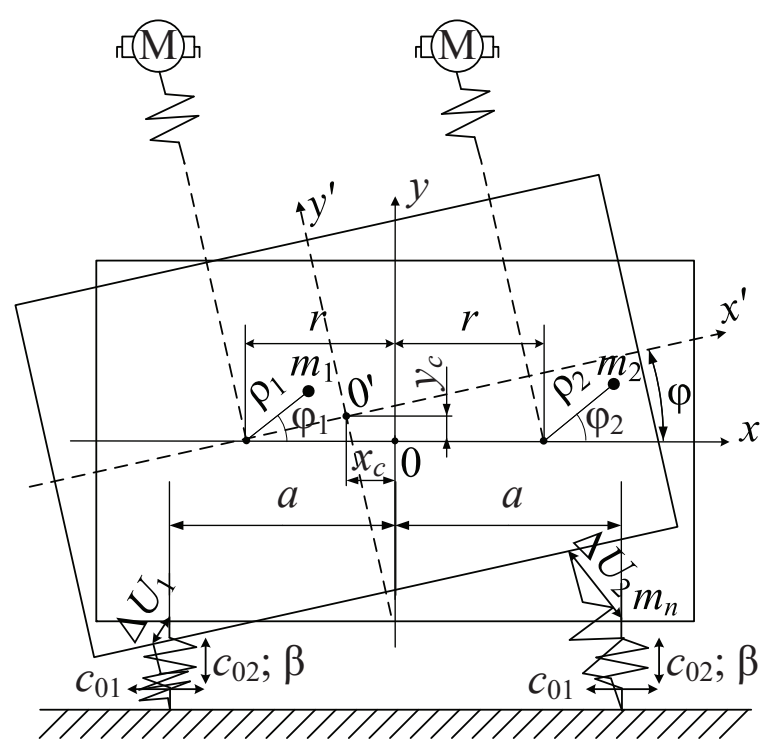

Figure 3. Two-rotor vibration unit with elastic cardan shafts.

the controlled plant not only the dynamics of drives, but also other features of the system that are important for practical implementation: the elasticity of the enginemechanism connection, which is expressed in the elasticity of cardan shafts connecting motors with unbalanced rotors, as well as the influence on the synchronization process of the use of observer estimates.

\section{Model of two-rotor vibration unit taking into ac- count the drive dynamics and the elasticity of the cardan shafts}

In this section the mathematical model of the unit SV-2 (Fig. 1) is described following [Tomchina, 2019]. Unlike [Tomchina, 2019], the elastic properties of the connection "executive motor-unbalanced rotor" are taken into account.

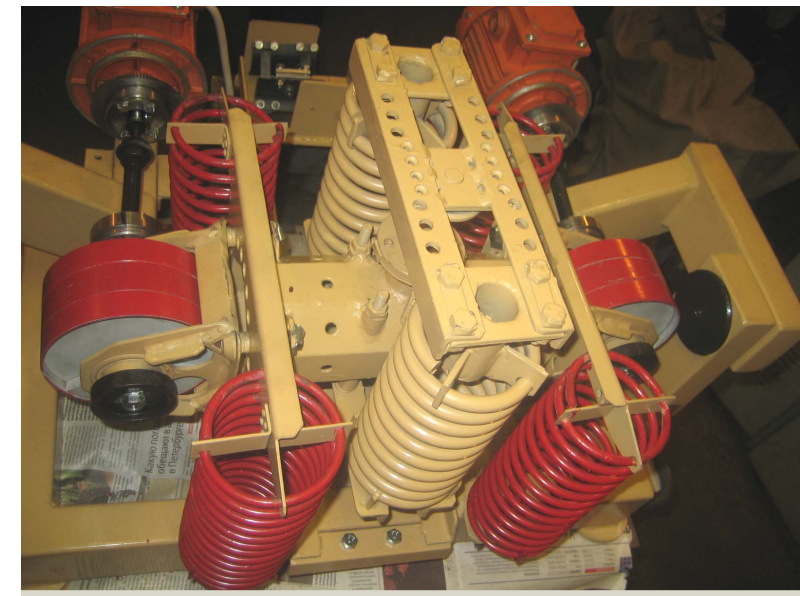

Figure 1. Vibration unit SV-2.

The unit consists of two rotors 4 installed on the vibrating platform 6 elastically connected with fixed basis 2,3 by springs 7 (Fig. 2).

Since the motors are located on motionless supports and the unbalanced rotors are mounted on the vibrating platform (bearings), the links implemented via cardan shafts should be considered as elastic ones.

The real mechanism is usually a system with distributed parameters. However in most cases it is affordable to consider the mechanism as a system with lumped parameters, while the following assumptions are generally accepted:

1. elastic links are weightless and characterized by constant rigidity, that is, a constant coefficient of proportionality between the torque (force) and deformation;

2. the forces and torques acting on the system are applied to point masses that are not deformed;

3. the distribution of deformation waves is not taken into account.

The kinematic diagram of the VU with elastic shafts between the motors and the rotors, schematically represented as springs connecting the motors $M$ and the attachment points of the rotors, is presented in Fig. 3. In this paper only torsional elasticity will be taken into account. The block scheme representing the elastic links of drive shafts with unbalanced rotors, may be presented in the following form (Fig. 4). The following notations are used in Fig. 3 and Fig. 4: $M_{m_{i}}, i=1,2$ are the electromechanical torques of motors; $\omega_{i}$ are the angular velocities of motors; $M_{r i}$ are the unbalanced rotor's own moments: $M_{r i}(t)=m \varrho g \cos \left(\varphi+\varphi_{i}\right) ; \tilde{M}_{s i}$ are the moments, caused by the platform influence:

$$
\begin{gathered}
\tilde{M}_{s i}=-\ddot{x}_{c} m \varrho \sin \left(\varphi+\varphi_{i}\right)+\ddot{y}_{c} m \varrho \cos \left(\varphi+\varphi_{i}\right)+ \\
\ddot{\varphi}\left(J_{i}+(-1)^{i} r m \varrho \cos \varphi_{i}\right)+(-1)^{i} \dot{\varphi}^{2} r m \varrho \sin \varphi_{i},
\end{gathered}
$$


$c_{B} \cdot b_{B}$ are the stiffness and damping coefficient of the cardan shafts, $\varphi, \varphi_{i}, i=1,2$ are angle of the support and rotation angles of the rotors, respectively, measured from the horizontal position, $x_{c}, y_{c}$ are the horizontal and vertical displacements of the vibrating platform center $\mathrm{O}^{\prime}$ from its equilibrium position $\mathrm{O}, m_{i}=m, i=1,2$ and $m_{n}$ are the masses of the rotors and supporting body, $J_{1}=J_{2}=J$ are the inertia moments of the rotors, $\varrho_{i}=\varrho, i=1,2$ are the rotor eccentricities, $c_{01}, c_{02}$ are the horizontal and vertical spring stiffness, $g$ is the gravity acceleration, $m_{0}$ is the total mass of the unit, $m_{0}=2 m+m_{n}, \beta$ is the damping coefficient, $k_{r}$ the friction coefficient in the bearings, the motor torques (controlling variables) $M_{i}=u_{i}(t)$ are calculated with algorithm (5), $r$ is the distance from the centers of the rotors to $\mathrm{O}^{\prime}$. It is assumed that the rotors are identical, rotor shafts are orthogonal to the motion of the support.

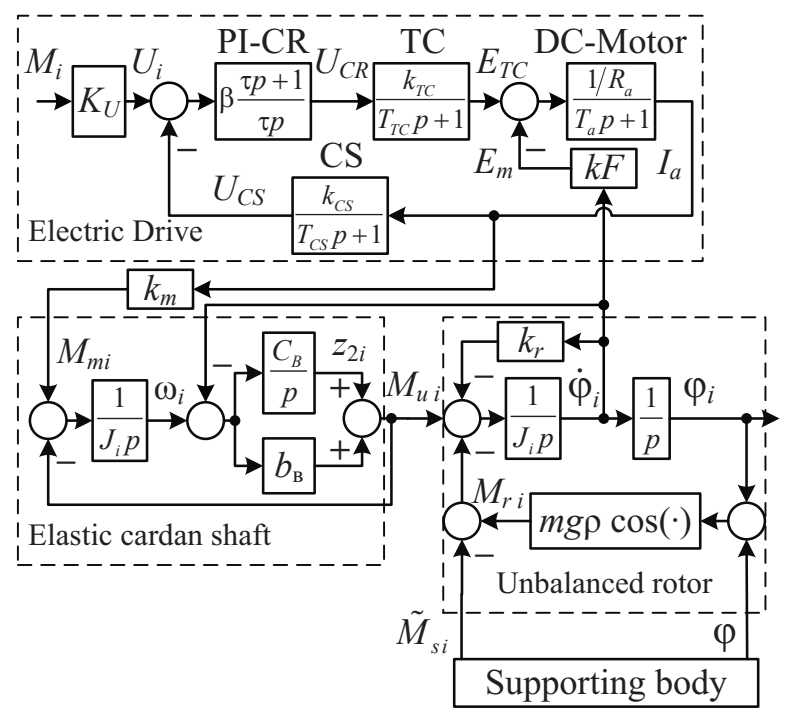

Figure 4. System engine - rotor taking into account the elasticity of the propeller shaft.

The equations of the mechanical part of VU dynamics have the following form [Tomchina, 2019]: $m_{0} \ddot{x}_{c}-\ddot{\varphi} m \varrho\left[\sin \left(\varphi+\varphi_{1}\right)+\sin \left(\varphi+\varphi_{2}\right)\right]-$ $\ddot{\varphi}_{1} m \varrho \sin \left(\varphi+\varphi_{1}\right)-\ddot{\varphi}_{2} m \varrho \sin \left(\varphi+\varphi_{2}\right)-$ $\dot{\varphi}^{2} m \varrho\left[\cos \left(\varphi+\varphi_{1}\right)+\cos \left(\varphi+\varphi_{2}\right)\right]-$ $\dot{\varphi}_{1}^{2} m \varrho \cos \left(\varphi+\varphi_{1}\right)-\dot{\varphi}_{2}^{2} m \varrho \cos \left(\varphi+\varphi_{2}\right)-$ $2 \dot{\varphi} \dot{\varphi}_{1} m \varrho \cos \left(\varphi+\varphi_{1}\right)-$

$2 \dot{\varphi} \dot{\varphi}_{2} m \varrho \cos \left(\varphi+\varphi_{2}\right)+2 c_{01} x_{c}+\beta \dot{x}_{c}=0 ;$ $m_{0} \ddot{y}_{c}+\ddot{\varphi} m \varrho\left[\cos \left(\varphi+\varphi_{1}\right)+\cos \left(\varphi+\varphi_{2}\right)\right]-$ $\ddot{\varphi}_{1} m \varrho \cos \left(\varphi+\varphi_{1}\right)-\ddot{\varphi}_{2} m \varrho \cos \left(\varphi+\varphi_{2}\right)-$ $\dot{\varphi}^{2} m \varrho\left[\sin \left(\varphi+\varphi_{1}\right)+\sin \left(\varphi+\varphi_{2}\right)\right]-$ $\dot{\varphi}_{1}^{2} m \varrho \sin \left(\varphi+\varphi_{1}\right)-\dot{\varphi}_{2}^{2} m \varrho \sin \left(\varphi+\varphi_{2}\right)-$ $2 \dot{\varphi} \dot{\varphi}_{1} m \varrho \sin \left(\varphi+\varphi_{1}\right)-2 \dot{\varphi} \dot{\varphi}_{2} m \varrho \sin \left(\varphi+\varphi_{2}\right)+$ $m_{0} g+2 c_{02} y_{c}+\beta \dot{y}_{c}=0$

$-\ddot{x}_{c} m \varrho\left[\sin \left(\varphi+\varphi_{1}\right)+\sin \left(\varphi+\varphi_{2}\right)\right]+$ $\ddot{y}_{c} m \varrho\left[\cos \left(\varphi+\varphi_{1}\right)+\cos \left(\varphi+\varphi_{2}\right)\right]+$ $\ddot{\varphi}\left[J+J_{1}+J_{2}-2 r m \varrho\left(\cos \varphi_{1}-\cos \varphi_{2}\right)\right]+$ $\ddot{\varphi}_{1}\left(J_{1}-r m \varrho \cos \varphi_{1}\right)+\ddot{\varphi}_{2}\left(J_{2}+r m \varrho \cos \varphi_{2}\right)+$ $\dot{\varphi}_{1}^{2} r m \varrho \sin \varphi_{1}-\dot{\varphi}_{2}^{2} r m \varrho \sin \varphi_{2}+2 r m \varrho \dot{\varphi} \dot{\varphi}_{1} \sin \varphi_{1}-$ $2 r m \varrho \dot{\varphi} \dot{\varphi}_{2} \sin \varphi_{2}+m \varrho g\left[\cos \left(\varphi+\varphi_{1}\right)+\right.$ $\left.\cos \left(\varphi+\varphi_{2}\right)\right]+c_{03} \varphi+\beta \dot{\varphi}=0 ;$ $-\ddot{x}_{c} m \varrho \sin \left(\varphi+\varphi_{1}\right)+\ddot{y}_{c} m \varrho \cos \left(\varphi+\varphi_{1}\right)+$ $\ddot{\varphi}\left(J_{1}-r m \varrho \cos \varphi_{1}\right)+\ddot{\varphi}_{1} J_{1}-\dot{\varphi}^{2} r m \varrho \sin \varphi_{1}+$ $m \varrho g \cos \left(\varphi+\varphi_{1}\right)+k_{c} \dot{\varphi}_{1}=M_{u 1}$;

$-\ddot{x}_{c} m \varrho \sin \left(\varphi+\varphi_{2}\right)+\ddot{y}_{c} m \varrho \cos \left(\varphi+\varphi_{2}\right)+$ $\ddot{\varphi}\left(J_{2}+r m \varrho \cos \varphi_{1}\right)+\ddot{\varphi}_{2} J_{2}+\dot{\varphi}^{2} r m \varrho \sin \varphi_{2}+$ $m \varrho g \cos \left(\varphi+\varphi_{2}\right)+k_{c} \dot{\varphi}_{2}=M_{u 2}$;

The "unbalanced rotor" structural diagram (Fig. 4) prepared in accordance with the fourth equation of system (1).

To convert the scheme into the state space equations, accounting elasticity, let us substitute the state variables $z_{1 i}=\omega_{i}$ and $z_{2 i}$ as the outputs of corresponding integrators $(i=1,2)$. Then the dynamics of the cardan shaft taking into account torsional elasticity are described by the differential equations:

$$
\begin{aligned}
& \dot{z}_{1 i}=\frac{1}{J_{i}}\left(M_{m i}-M_{u i}\right)= \\
& =\frac{1}{J_{i}} M_{m i}-\frac{1}{J_{i}}\left(z_{2 i}+b_{B}\left(z_{1 i}-\dot{\varphi}_{i}\right)\right), \\
& \dot{z}_{2 i}=c_{B}\left(z_{1 i}-\dot{\varphi}_{i}\right) \quad M_{u i}=z_{2 i}+b_{B}\left(z_{1 i}-\dot{\varphi}\right)
\end{aligned}
$$

When taking into account the dynamics of the drive in the simulation process the control torque $M_{m 1}$ arriving at the input of the "elastic cardan shaft" is formed in accordance with the structural diagram of the "electric drive". Since the laboratory setup SV-2 used the DC motors, the electric drive structure is selected as the traditional single-circuit system with current loop and proportional-integral (PI) current controller $W_{C R}(p)=$ $b(\tau p+1) / \tau p$ is configured to optimum modulo; $b, \tau$ are dynamic gain and time constant of the regulator. The following notation is used here: CR is the current regulator; TC is the power (thyristor type) converter; CS is the current sensor; $I_{a}$ is the armature current; $E_{T C}$ and $E_{m}$ are converter and motor EMFs; $k_{T C}$ and $k_{C S}$ are converter and current feedback gains; $k F$ is the motor torque (EMF) coefficient; $T_{T C}$ and $T_{C S}$ are converter and current sensor time constants; $T_{a}$ is the armature time constant; $R_{a}$ is the armature curcuit resistance; $U_{C R}$ and $U_{C S}$ are current controller and current sensor output voltages: $U$ is the voltage corresponding to the 
motor torque $M_{m i}$ determined by the value $M_{i}$, calculated in the algorithm and obeys a system of differential and algebraic equations (index " $i$ " is omitted for simplicity):

$$
\begin{array}{r}
\dot{I}_{a}=\frac{1}{T_{a}}\left(-I_{a}+\frac{1}{R_{a}}\left(E_{T C}-E_{m}\right)\right), \\
\dot{E}_{T C}=\frac{1}{T_{T C}}\left(-E_{T C}+k_{T C} U_{C R}\right), \\
\dot{U}_{C S}=\frac{1}{T_{C S}}\left(-U_{C S}+k_{C S} I_{a}\right), \\
\dot{U}_{C S i}=\frac{b}{\tau}\left(U_{i}-U_{C S}\right), \\
U_{C S}=b\left(U_{i}-U_{C S}\right)+U_{C S}, \\
E_{m}=k F \dot{\varphi}, \quad M_{m}=k_{M} I_{a}
\end{array}
$$

\section{Speed-Gradient Control Algorithms with time- varying observers for synchronization of two- rotor vibration unit}

The problem of maintaining a stable synchronous mode of rotation of unbalanced rotors of a two-rotor VU for a model that takes into account the dynamics of electric drives, using observer estimates in the control algorithm, was considered in [Tomchina, 2020]. Algorithm [Tomchina, 2020] can also be applied when solving the problem of controlling the vibration field of a two-rotor VU.

To provide a synchronous rotation mode of unbalanced rotors for two-rotor VU, it is suggested to use speed- gradient method with an objective functional in the following form:

$$
Q(z)=0.5\left\{(1-\alpha)\left(H-H^{*}\right)^{2}+\alpha\left(\dot{\varphi}_{1} \pm \dot{\varphi}_{2}\right)^{2}\right\},
$$

where $z=\left[x_{c}, \dot{x}_{c}, y_{c}, \dot{y}_{c}, \varphi, \dot{\varphi}, \varphi_{1}, \dot{\varphi}_{1}, \varphi_{2}, \dot{\varphi}_{2}\right]^{T}, 0<$ $\alpha<1$ is weight coefficient; $H$ is total mechanical energy of a system (1), $H^{*}$ is the desired value of $H$.

Upon reaching the control goal (4) i.e. $Q(z)=0$, we get $H=H^{*}$ and equal rotor speeds $\dot{\varphi}_{1}=\dot{\varphi}_{2}$. This approach, when choosing a target functional that uses information only about the energy of the mechanical part of the $\mathrm{VU}$, is based on the properties of the speed gradient method [Firsova, 2002]. In particular, the algorithms synthesized using the velocity gradient method provide dissipativity in the case of fast-flowing structural disturbances, which in this work include processes caused by the dynamics of electric drives and elastic vibrations of cardan shafts.

The proportional-integral (PI-) speed-gradient algorithm in the finite form with the objective functional (4) is as follows [Tomchina, 2019]:

$$
\begin{aligned}
M_{1}= & -\gamma_{1}\left\{(1-\alpha)\left(H-H^{*}\right) \dot{\varphi}_{1}+\right. \\
& \left.\frac{\alpha}{J_{1}}\left(\dot{\varphi}_{1} \pm \dot{\varphi}_{2}\right)+\frac{\alpha}{J_{1}}\left(\varphi_{1} \pm \varphi_{2}+\Delta \varphi_{1}^{*}\right)\right\} \\
M_{2}= & -\gamma_{2}\left\{(1-\alpha)\left(H-H^{*}\right) \dot{\varphi}_{2} \pm\right. \\
& \left.\frac{\alpha}{J_{2}}\left(\dot{\varphi}_{1} \pm \dot{\varphi}_{2}\right) \pm \frac{\alpha}{J_{2}}\left(\varphi_{1} \pm \varphi_{2}+\Delta \varphi_{2}^{*}\right)\right\}
\end{aligned}
$$


where $\Delta \varphi_{1}^{*}, \Delta \varphi_{2}^{*}$ are some constant phase shift values, $\gamma_{i}>0$.

As it was shown in [Tomchina, 2020; Fradkov et al., 2013], it is the setting of certain values of the parameters of the algorithm $\Delta \varphi_{1}^{*}, \Delta \varphi_{2}^{*}$ that makes it possible to obtain a different type of VU vibration fields.

Total mechanical energy of a system (1) $H=T+$ $\Pi$, where kinetic and potential energies $T$ and $\Pi$ are as follows:

$$
\begin{aligned}
& T=0.5 m_{0}\left(\dot{x}_{c}^{2}+\dot{y}_{c}^{2}\right)+0.5 \dot{\varphi}^{2}\left(J+J_{1}+J_{2}-\right. \\
& \left.2 r m \varrho\left(\cos \varphi_{1}-\cos \varphi_{2}\right)\right)+0.5 J_{1} \dot{\varphi}_{1}^{2}+0.5 J_{2} \dot{\varphi}_{2}^{2}+ \\
& \dot{\varphi} \dot{\varphi}_{1}\left(J_{1}-r m \varrho \cos \varphi_{1}\right)+\dot{\varphi}_{2}\left(J_{2}+r m \varrho \cos \varphi_{2}\right)- \\
& \dot{x}_{c} \dot{\varphi} m \varrho\left(\sin \left(\varphi+\varphi_{1}\right)+\sin \left(\varphi+\varphi_{2}\right)\right)+ \\
& \dot{y}_{c} \dot{\varphi} m \varrho\left(\cos \left(\varphi+\varphi_{1}\right)+\cos \left(\varphi+\varphi_{2}\right)\right)- \\
& \dot{x}_{c} \dot{\varphi}_{1} m \varrho \sin \left(\varphi+\varphi_{1}\right)+\dot{y}_{c} \dot{\varphi}_{1} m \varrho \cos \left(\varphi+\varphi_{1}\right)- \\
& \dot{x}_{c} \dot{\varphi}_{2} m \varrho \sin \left(\varphi+\varphi_{2}\right)+\dot{y}_{c} \dot{\varphi}_{2} m \varrho \cos \left(\varphi+\varphi_{2}\right), \\
& \Pi=m_{0} g y_{c}+m \varrho\left(\sin \left(\varphi+\varphi_{1}\right)+\sin \left(\varphi+\varphi_{2}\right)\right)+ \\
& c_{01}\left(x_{c}^{2}+\alpha^{2} \cos ^{2} \varphi\right)^{2}+c_{02}\left(y_{c}^{2}+\alpha^{2} \sin ^{2} \varphi\right)^{2},
\end{aligned}
$$

Also, taking into account the properties of the speed gradient algorithms, we simplify the expression for the total mechanical energy $H$ in order to exclude the variables that cannot be measured by existing sensors and are sufficiently small to be neglected. In [Fradkov et al., 2013] the possibility of effective control in the absence of information about the platform rotation angle $\varphi$ was shown. Therefore, it is possible to simplify the expression for the total energy by setting $\varphi=0$. In addition the horizontal movements of the platform $x_{c}$ are neglectable too.

But even with such a simplification of the expression for the total mechanical energy, information is not enough: only the phase and angular velocity of the rotors, measured with an encoder, and the vertical position of the platform $y(t)$ are available for measurement. However, the speed of vertical movements $\dot{y}_{c}$ cannot be ignored and will be restored by the observer. To justify such a simplification a series of computer simulations will be performed.

When synthesizing the observer in [Tomchina, 2020], a simplified model of the VU dynamics was used which did not take into account the displacement along the horizontal axis $x_{c}$ and the angle $\varphi$ of the platform rotation in the vertical plane. As a basic equation for constructing an observer, the second equation of system (1) with the indicated simplifications was considered, which was represented in the form

$$
m_{0} \ddot{y}_{c}+\beta \dot{y}_{c}+2 c_{0} y_{c}=F(t)
$$

where

$$
\begin{aligned}
& F(t)=-m \varrho \sin \varphi_{1} \ddot{\varphi}_{1}-m \varrho \cos \varphi_{1} \dot{\varphi}_{1}^{2}- \\
& m \varrho \sin \varphi_{2} \ddot{\varphi}_{2}-m \varrho \cos \varphi_{2} \dot{\varphi}_{2}^{2}-m_{0} g .
\end{aligned}
$$

Further, from the indicated equations of the dynamics of the VU, expressions for were found, which were substituted into expression (7), after which equation (7) was presented in the form

$$
\begin{aligned}
& m_{0} \ddot{y}_{c}+\beta_{1}(t) \dot{y}_{c}+2 c_{0} y_{c}=F_{1}(t), \\
& \beta_{1}(t)=\beta \frac{m_{0} J_{1} J_{2}}{m_{0} J_{1} J_{2}-J_{1} m^{2} \varrho^{2} \sin ^{2} \varphi_{2}-J_{2} m^{2} \varrho^{2} \sin ^{2} \varphi_{1}}= \\
& =\beta \frac{m_{0} J_{1} J_{2}}{\Delta(t)},
\end{aligned}
$$

where

$$
\begin{aligned}
F_{1}(t) & =F(t)+\left(\frac{J_{2} m^{2} \varrho^{2} \sin ^{2} \varphi_{1}}{\Delta(t)}+\right. \\
& \left.+\frac{J_{1} m^{2} \varrho^{2} \sin ^{2} \varphi_{2}}{\Delta(t)}\right) \beta \dot{y}_{c} .
\end{aligned}
$$

After that the following constraints were introduced

$$
x_{1}=y_{c}, \quad x_{2}=\dot{y}_{c}
$$

and equation (9) was converted into the equation in the state space

$$
\left\{\begin{array}{l}
\dot{x}_{1}(t)=x_{2} ; \\
\dot{x}_{2}(t)=-\beta_{0}(t) x_{2}(t)-c_{0}^{\prime} x_{1}(t)+F_{2}(t),
\end{array}\right.
$$

where $\beta_{0}(t)=\beta_{1}(t) / m_{0} ; c_{0}^{\prime}=2 c_{0} / m_{0} ; F_{2}(t)=$ $F_{1}(t) / m_{0}$.

Then the equations of the non-stationary observer of full order are written in the form:

$$
\left\{\begin{aligned}
\dot{\hat{x}}_{1}(t) & =\hat{x}_{2}(t)+k_{1}\left(x_{1}(t)-\hat{x}_{1}(t)\right) ; \\
\hat{\hat{x}}_{2}(t) & =-\beta_{0}(t) \hat{x}_{2}(t)-c_{0}^{\prime} \hat{x}_{1}(t)+F_{2}(t)+ \\
& +k_{2}\left(x_{1}(t)-\hat{x}_{1}(t)\right)
\end{aligned}\right.
$$

where $x_{1}(t)$ is measured by sensors.

The observer-based algorithm

$$
\begin{aligned}
M_{1}= & -\gamma_{1}\left\{(1-\alpha)\left(\hat{H}-H^{*}\right) \dot{\varphi}_{1}+\right. \\
& \left.\frac{\alpha}{J_{1}}\left(\dot{\varphi}_{1} \pm \dot{\varphi}_{2}\right)+\frac{\alpha}{J_{1}}\left(\varphi_{1} \pm \varphi_{2}+\Delta \varphi_{1}^{*}\right)\right\} ; \\
M_{2}= & -\gamma_{2}\left\{(1-\alpha)\left(\hat{H}-H^{*}\right) \dot{\varphi}_{2} \pm\right. \\
& \left.\frac{\alpha}{J_{2}}\left(\dot{\varphi}_{1} \pm \dot{\varphi}_{2}\right) \pm \frac{\alpha}{J_{2}}\left(\varphi_{1} \pm \varphi_{2}+\Delta \varphi_{2}^{*}\right)\right\} .
\end{aligned}
$$

where $\hat{\dot{y}}_{c}=\hat{x}_{2}$ is obtained from observer (11),

$$
\begin{aligned}
& \hat{H}=0.5 m_{0} \hat{\dot{y}}_{c}^{2}+0.5 J_{1} \dot{\varphi}_{1}^{2}+0.5 J_{2} \dot{\varphi}_{2}^{2}+ \\
& +\hat{\dot{y}}_{c} \dot{\varphi}_{1} m \varrho \cos \varphi_{1}+\dot{\dot{y}}_{c} \dot{\varphi}_{2} m \varrho \cos \varphi_{2}+ \\
& +m_{0} g y_{c}+m \varrho g\left(\sin \varphi_{1}+\sin \varphi_{2}\right)+c_{02} y_{c}^{2}
\end{aligned}
$$

The shape of vibration fields obtained when using the synchronization algorithm with an observer (12) is investigated using simulation in the MATLAB environment. 
Table 1.

\begin{tabular}{|c|c|c|c|c|c|}
\hline \multicolumn{2}{|c|}{$\begin{array}{c}\text { Observer } \\
\text { gains }\end{array}$} & \multicolumn{2}{c|}{$\begin{array}{c}\text { Maximum value } \\
\text { of observation } \\
\text { errors (no } \\
\text { saturation) }\end{array}$} & \multicolumn{2}{c|}{$\begin{array}{c}\text { Maximum value } \\
\text { of observation } \\
\text { errors (saturation } \\
\text { level 5 N.m) }\end{array}$} \\
\hline$k_{1}$ & $k_{2}$ & $e_{1}, \mathrm{~m}$ & $e_{2}, \mathrm{~m} / \mathrm{s}$ & $e_{1}, \mathrm{~m}$ & $e_{2}, \mathrm{~m} / \mathrm{s}$ \\
\hline 1 & 10 & 0.0068 & 0.187 & 0.0075 & 0.198 \\
\hline 1 & 100 & 0.0062 & 0.170 & 0.0054 & 0.130 \\
\hline 10 & 100 & 0.0038 & 0.146 & 0.0038 & 0.101 \\
\hline 10 & 1000 & 0.0026 & 0.122 & 0.00178 & 0.079 \\
\hline 10 & 10000 & 0.0015 & 0.177 & 0.0016 & 0.179 \\
\hline 100 & 100 & 0.003 & 0.338 & 0.0026 & 0.286 \\
\hline 100 & 1000 & 0.0017 & 0.210 & 0.0015 & 0.186 \\
\hline
\end{tabular}

\section{Simulation results}

Earlier, the study of the shape of vibration fields for a two-rotor VU was carried out for a model that takes into account only the dynamics of the mechanical part of the unit [Firsova, 2002] and for a model in which dynamics of electric drives are taken into account [Tomchina, 2019].

In this paper we consider the influence of the following three factors on the shape of vibration fields:

saturation restrictions for the controlling torques $M_{i}$ (12) of the engine;

the use of observer estimates when calculating control moments;

the influence of the elasticity of the universal joint shafts connecting the motors and unbalanced rotors.

All three factors are essential in the practical implementation of control algorithms. First of all, the influence of level constraints for the electromechanical moments of the engine was investigated when using the observer's estimates when calculating the controlling torques (12).

Table 1 presents quantitative indicators for the maximum values of observation errors for the case of calculating the torques with and without saturation. The observation errors are denoted as $e_{1}, e_{2}$.

As can be seen from the table, the introduction of saturation on the control torques (12) does not have a significant effect on the amplitude of observation errors, but without deteriorating the quality of control it can significantly limit the value of start up currents and EMF of thyristor convertor (Fig. 5, Fig. 6). In addition, as can be seen from the table, the best results were obtained with the values of the amplification factors $k_{1}=100$, $k_{2}=1000\left(e_{1}=1 \cdot 10^{-4} \mathrm{~m}, e_{2}=0.01 \mathrm{~m} / \mathrm{s}\right)$. It should be noted that in addition to the fact that good assessment indicators are obtained with large coefficients $k_{1}$ and $k_{2}$, the ratio between these coefficients is also important. So, for $k_{1}=10, k_{2}=10000$, the processes are the worst in terms of observation errors.
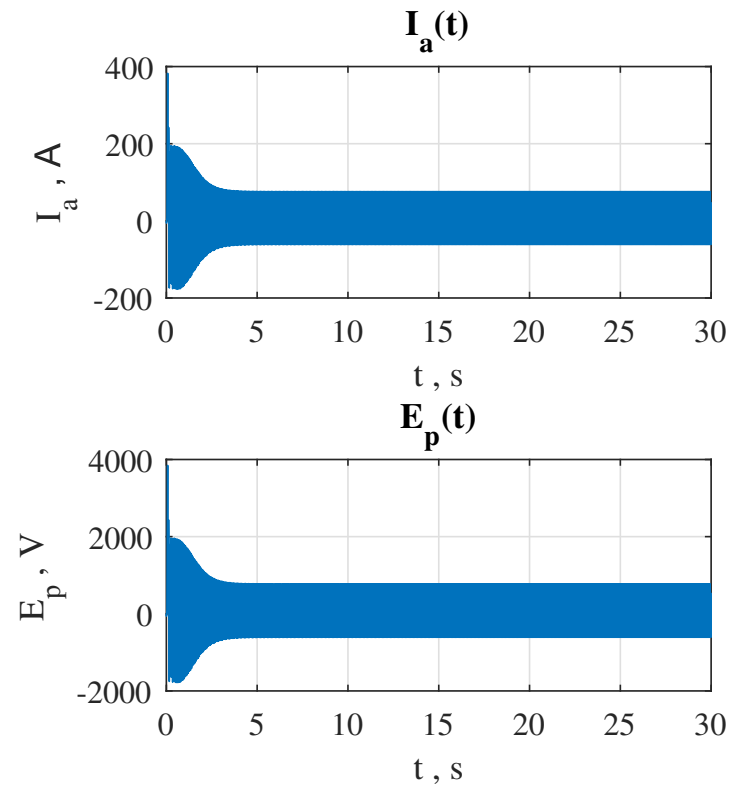

Figure 5. Investigation of the dynamics of a model of a two-rotor vibration plant with varying observer coefficients without taking into account the constraint
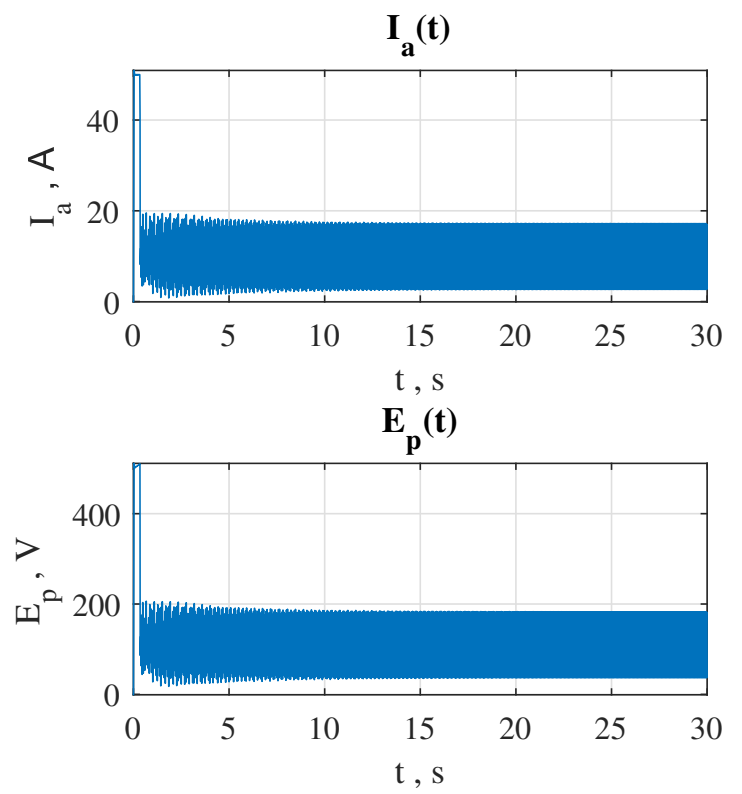

Figure 6. Investigation of the dynamics of a model of a two-rotor vibration unit with varying observer coefficients for $\left|M_{i}\right| \leq 5 \mathrm{~N} \cdot \mathrm{m}$. 
Since the steady-state phase shift determines the shape of the vibration field (that is, the trajectories of steadystate oscillations for different points of the bearing platform), it is necessary to find out the dependence of the steady-state phase shift $\Delta \varphi$ on $\Delta \varphi_{1}^{*}$ specified in the synchronization algorithm (we assume that $\Delta \varphi_{2}^{*}=0$ ), and, as shown by modeling, this dependence is different for different desired values for total energy $H^{*}$.

The table of the dependence of the indicated skews with varying the observer gains presented in Table 2 , and the corresponding nomograms of the dependence of the steady-state phase shift $\Delta \varphi$ on $\Delta \varphi_{1}^{*}$ are shown in Fig. 7.

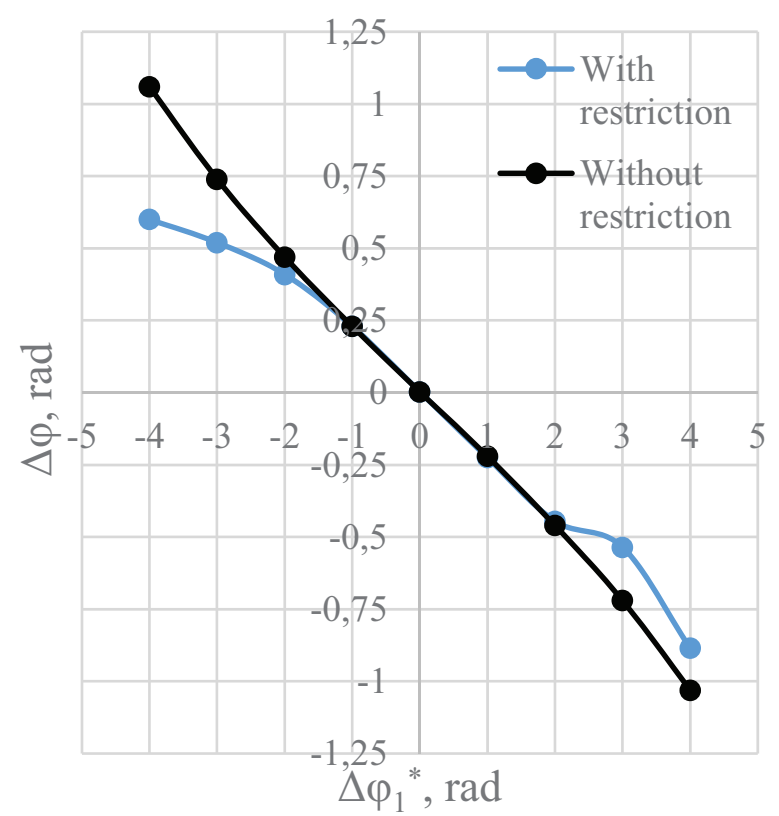

Figure 7. Nomograms of dependence $\Delta \varphi, \operatorname{rad}$ on $\Delta \varphi_{1}^{*}, \operatorname{rad}$ (yellow line — with saturation, black line — no saturation)

As can be seen from the table and nomograms, the values of the observer's coefficients do not affect the values of the steady-state phase shifts $\Delta \varphi$. Moreover, in the absence of restrictions on the controlling torques, the indicated values of $\Delta \varphi$ for a system with an observer and a system without an observer coincide. This can be explained by the fact that the decay time of the observation processes is higher than the synchronization time. However, as shown by modeling, the magnitude of the limitation on the control torques affects the appearance of the nomograms. On the other hand, in the main working interval of the phase shift $\Delta \varphi_{1}^{*}$, that is, in the interval $(-\pi / 2, \pi / 2)$, the lines in the figure are close. It means that the steady-state phase shift $\Delta \varphi$, which determines the slope of the trajectory axes of the platform points, in the presence of constraints, is the same as in the case of a system with unlimited control torques.

Further, the study of vibration fields for the model of a two-rotor VU which took into account the dynamics of the electric drive, the limitation of the control torques and the elasticity of the universal joint shafts connecting the motors and unbalanced rotors was carried out. In the course of computer simulation, the parameters of the elastic connection were varied: the stiffness coefficient $c_{B}$ and the damping coefficient $b_{B}$. The results are given in Table 3. They were obtained for a given total energy $H^{*}=150 \mathrm{~J}$ and a constraint equal to $\left|M_{i}\right| \leq 5 \mathrm{~N} \cdot \mathrm{m}$. The table shows the following indicators of the efficiency of synchronous mode control: $t_{\mathrm{syn}}, \mathrm{s}-$ synchronization time, $t_{\mathrm{tr}}, \mathrm{s}-$ transient process time, $\left|y(t)_{\max }\right|, \mathrm{m}-$ maximum amplitude of platform oscillations, $\left|M_{u_{\max }}\right|$, $\mathrm{Nm}$ - maximum values elastic moment.

Nomograms of the dependence of the steady-state phase shift $\Delta \varphi$ on $\Delta \varphi_{1}^{*}$, for various parameters of the elastic shaft are shown in Fig. 8. Fig. 9 shows nomograms reflecting the indicated dependence at a fixed value of the stiffness coefficient $c_{B}=1000 \mathrm{Nm} / \mathrm{rad}$ and varying the parameter $b_{B}$.

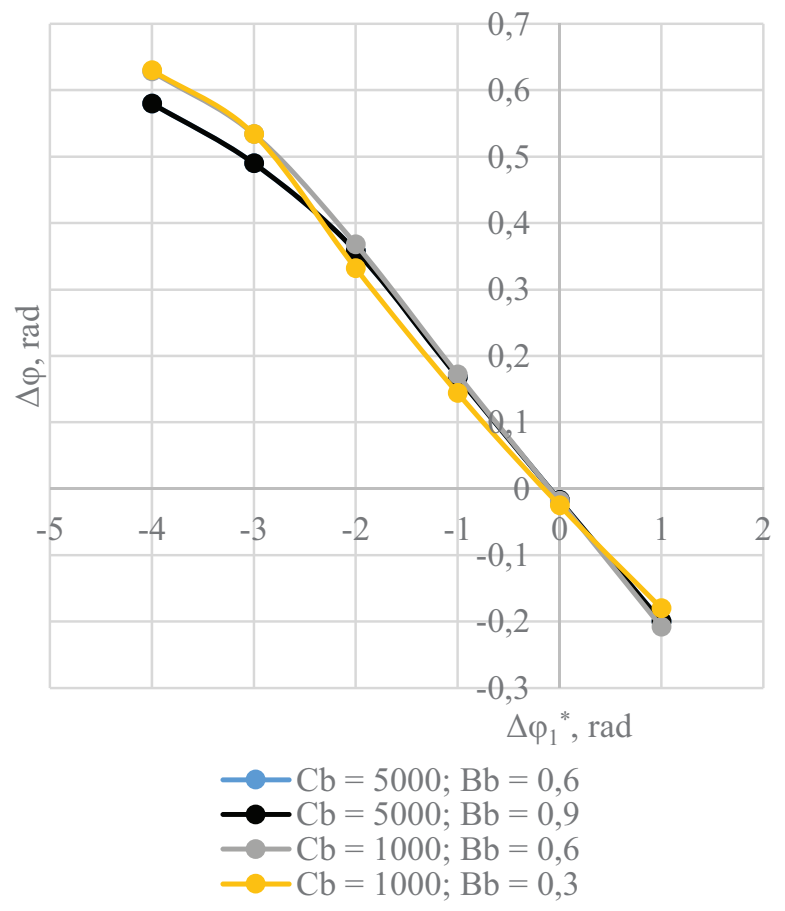

Figure 8. Nomograms of the dependence of the steady-state phase shift $\Delta \varphi$, rad, on $\Delta \varphi_{1}^{*}$,rad, for various parameters of the elastic shaft

As noted above, a feature of two-rotor VUs is the complex nature of platform oscillations: different points of the platform move along different trajectories, which leads to the formation of different vibration fields. The figures below show the projections of the trajectories of various points of the platform onto the vertical plane which form the vibration field.

In [Tomchina, 2018], the construction of a vibration field for a VU with two vibration actuators was consid- 
Table 2.

\begin{tabular}{|c|c|c|c|c|c|c|}
\hline \multirow[b]{2}{*}{$\begin{array}{c}\Delta \varphi_{1}^{*}, \\
\operatorname{rad}\end{array}$} & \multicolumn{2}{|c|}{$k_{1}=10, k_{2}=10$} & \multicolumn{2}{|c|}{$k_{1}=100, k_{2}=1000$} & \multicolumn{2}{|c|}{ No observer } \\
\hline & $\begin{array}{c}\Delta \varphi, \mathrm{rad}, \text { for } \\
\left|M_{i}\right| \leq 5 \mathrm{~N} \cdot \mathrm{m}\end{array}$ & $\begin{array}{c}\Delta \varphi, \text { rad, no } \\
\text { saturation }\end{array}$ & $\begin{array}{c}\Delta \varphi, \text { rad, for } \\
\left|M_{i}\right| \leq 5 \mathrm{~N} \cdot \mathrm{m}\end{array}$ & $\begin{array}{c}\Delta \varphi, \text { rad, no } \\
\text { saturation }\end{array}$ & $\begin{array}{c}\Delta \varphi, \mathrm{rad}, \text { for } \\
\left|M_{i}\right| \leq 5 \mathrm{~N} \cdot \mathrm{m}\end{array}$ & $\begin{array}{c}\Delta \varphi, \text { rad, no } \\
\text { saturation }\end{array}$ \\
\hline-4 & 0.6 & 1.06 & 0.6 & 1.06 & 0.6 & 1.06 \\
\hline-3 & 0.52 & 0.74 & 0.52 & 0.74 & 0.52 & 0.74 \\
\hline-2 & 0.41 & 0.47 & 0.41 & 0.47 & 0.41 & 0.47 \\
\hline-1 & 0.23 & 0.23 & 0.23 & 0.23 & 0.23 & 0.23 \\
\hline 0 & 0.003 & 0.003 & 0.003 & 0.003 & 0.003 & 0.003 \\
\hline 1 & -0.225 & -0.22 & -0.225 & -0.22 & -0.225 & -0.22 \\
\hline 2 & -0.445 & -0.46 & -0.445 & -0.46 & -0.445 & -0.46 \\
\hline 3 & -0.535 & -0.72 & -0.535 & -0.72 & -0.535 & -0.72 \\
\hline 4 & -0.885 & -1.03 & -0.885 & -1.03 & -0.885 & -1.03 \\
\hline
\end{tabular}

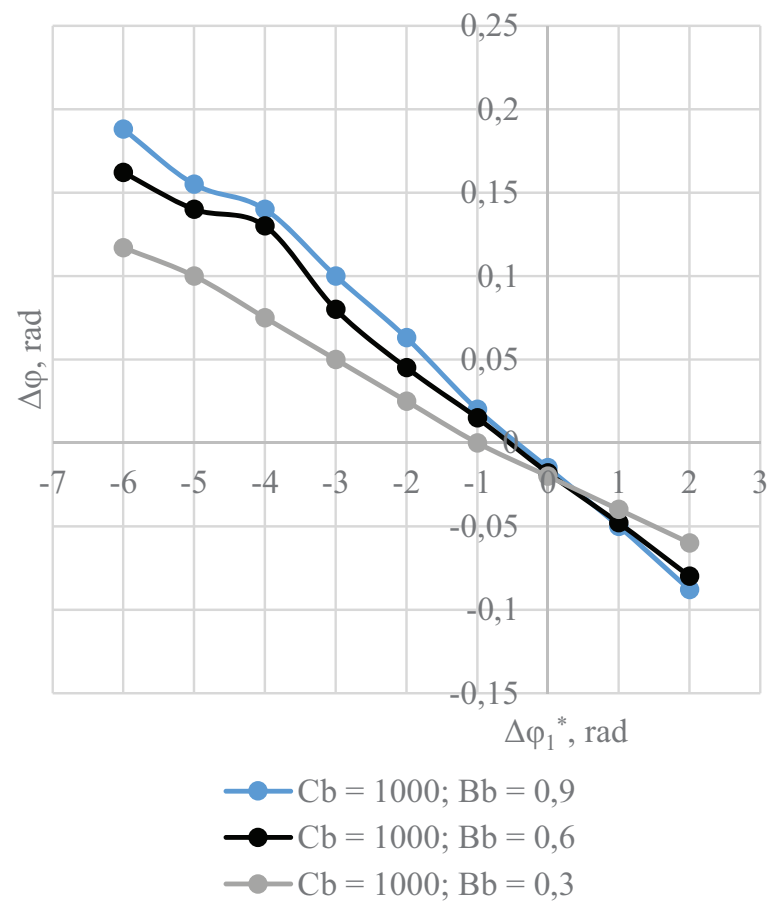

Figure 9. Nomograms of the dependence of the steady-state phase shift $\Delta \varphi$, rad, on $\Delta \varphi_{1}^{*}$,rad, for $c_{B}=1000 \mathrm{Nm} / \mathrm{rad}$ and varyng the parameter $b_{B}$.
Table 3.

\begin{tabular}{|c|c|c|c|c|}
\hline $\begin{array}{c}c_{B}, \mathrm{Nm} / \mathrm{rad} \\
b_{B}, \mathrm{~N} \cdot \mathrm{m} \cdot \mathrm{s}\end{array}$ & $t_{\mathrm{syn}, \mathrm{s}}$ & $t_{\mathrm{tr}, \mathrm{s}}$ & $\begin{array}{c}\left|y(t)_{\max }\right|, \\
\mathrm{m}\end{array}$ & $\begin{array}{c}\left|M_{u_{\max }}\right|, \\
\mathrm{N} \cdot \mathrm{m}\end{array}$ \\
\hline $\begin{array}{c}c_{B}=500 \\
b_{B}=0.8\end{array}$ & 1.288 & 0.73 & 0.057 & 9.8 \\
\hline $\begin{array}{c}c_{B}=500 \\
b_{B}=0.5\end{array}$ & 1.722 & 0.729 & 0.057 & 10.32 \\
\hline $\begin{array}{c}c_{B}=1000 \\
b_{B}=0.4\end{array}$ & 1.225 & 0.725 & 0.057 & 9.04 \\
\hline $\begin{array}{c}c_{B}=1000 \\
b_{B}=0.8\end{array}$ & 2.593 & 0.727 & 0.057 & 8.95 \\
\hline $\begin{array}{c}c_{B}=2000 \\
b_{B}=0.7\end{array}$ & 1.181 & 0.715 & 0.057 & 8.3 \\
\hline $\begin{array}{c}c_{B}=2500 \\
b_{B}=0.5\end{array}$ & 1.179 & 0.726 & 0.057 & 8.1 \\
\hline $\begin{array}{c}c_{B}=3000 \\
b_{B}=0.9\end{array}$ & 1.177 & 0.7 & 0.057 & 8.09 \\
\hline $\begin{array}{c}c_{B}=3000 \\
b_{B}=0.4\end{array}$ & 2.591 & 0.723 & 0.057 & 8.06 \\
\hline $\begin{array}{c}c_{B}=5000 \\
b_{B}=0.6\end{array}$ & 1.173 & 0.733 & 0.057 & 7.90 \\
\hline $\begin{array}{c}c_{B}=5000 \\
b_{B}=0.3\end{array}$ & 2.429 & 0.723 & 0.057 & 7.94 \\
\hline
\end{tabular}

ered and a Universal Field Diagram) (UFD) was presented, which is most effective for vibration transport and allows one to eliminate blockages at the unloading end of the platform. 
rad in algorithm (12) is shown. In accordance with the nomograms (Fig. 8), the steady-state phase shift was $\Delta \varphi=0.016 \mathrm{rad}$, which provided an almost uniform vibration field.

The field diagram shown in Fig. 10 can be considered as the best for vibration transportation of bulk materials, if we consider the left end of the platform as the loading end, and the right end as the unloading end. However, as is seen in Fig. 12, the use of algorithm (12) to control the type of the vibration field can provide any inclinations of the axes of the corresponding elliptical trajectories. Thus, the presented nomograms make it possible to select the parameters of the control algorithm that ensure the optimization of work processes.

\section{Conclusion}

Based on the study of the proposed algorithm for controlling the vibration field of a mechatronic double-rotor unit for sieving bulk materials, the following conclusions can be drawn.

The rotor synchronization control algorithm synthesized for the model of a two-rotor VU, taking into account the elasticity of cardan shafts and the dynamics of electric drives, using estimates of a non-stationary observer, makes it possible to control the steady-state phase shift of the rotors by varying the phase shift specified in the algorithm and the given energy within wide limits. The control algorithm provides both an approximate frequency synchronization and an approximate coordinate synchronization.

As shown by the simulation of the VU dynamics described by equations (1-3), when using the observerbased synchronization algorithm, taking into account the saturation of the control torques, the transient time for the rotor speeds is practically independent of the parameters of the elastic shafts, and the synchronization time can increase with a decrease in the damping coefficient.

The use of observer estimates in the control algorithm (12) does not distort the form of vibration fields. The nomograms constructed under control using algorithms (5) and (12) at coincide.

Since the type of the vibration field at fixed coordinates of the attachment points of the rotors and at constant mass-inertial parameters of the unbalances is determined by the phase shift of the rotors $\Delta \varphi$, then according to the nomograms of the dependence of $\Delta \varphi$ on $\Delta \varphi_{1}^{*}$ constructed in the work, it is possible to provide the desired form of the vibration field, depending on the level of limitation on the control torques and the values of the stiffness coefficient and the damping coefficient of propeller shafts.

Future research may be aimed at the employing adaptive observers allowing one to improve control performance under conditions of strong uncertainty [Efinov and Fradkov, 2015] and implementing of the ideas of control of actuators with internal degrees of freedom [Fradkov et al., 2005].

\section{References}

Andrievsky, B. R. and Fradkov, A. L. (2021). Speed gradient method and its applications. Automation and Remote Control, 82 (9), pp. 1463-1518.

Chen, B., Yan, J., Yin, Z., and Tamma, K. K. (2021). A new study on dynamic adjustment of vibration direction angle for dual-motor-driven vibrating screen. Proceedings of the Institution of Mechanical Engineers, Part E: Journal of Process Mechanical Engineering, 235 (2), pp. 186-196.

Efinov, D. and Fradkov, A. (2015). Design of impulsive adaptive observers for improvement of persistency of excitation. International Journal of Adaptive Control and Signal Processing, 29 (6), pp. 765-782.

el.al., I. B. (2001). Determination of the field of trajectories of points of the body of a vibration machine with two unbalanced vibration actuators. Obogaschenie Rud, (2), pp. 39-42.

Firsova, A. D. (2002). Vibration fields generated by unbalanced vibration actuators. In Collection of reports of the 5th International Conference on Problems of Oscillations (ICOVP-2001). Moscow: IMash, pp. 487491.

Fradkov, A., Andrievsky, B., and Boikov, K. (2005). Control of the coupled double pendulums system. Mechatronics, 15 (10), pp. 1289-1303.

Fradkov, A. L. (1980). Speed-gradient scheme and its application in adaptive control problems. Automation and Remote Control, 40 (9), pp. 1333-1342.

Fradkov, A. L., Tomchina, O. P., Andrievsky, B., and Boikov, V. I. (2021). Control of phase shift in tworotor vibration units. IEEE Transactions on Control Systems Technology, 29(3), pp. 1316-1322.

Fradkov, A. L., Tomchina, O. P., Galitskaya, V. A., and Gorlatov, D. V. (2013). Integral differentiating speedgradient algorithms in multiple synchronization issues for vibration units. Scientific and Technical Journal of Information Technologies, Mechanics and Optics, 29(1), pp. 30-37.

Gouskov, A., Panovko, G., and Shokhin, A. (2021). To the issue of control resonant oscillations of a vibrating machine with two self-synchronizing inertial exciters. Lecture Notes in Mechanical Engineering, 58, pp. 515-526.

Tomchina, O. (2018). Control of vibrational field in a cyberphysical vibration unit. Cybernetics and Physics, 7 (3), pp. 144-151.

Tomchina, O. (2019). Control of vibrational field in a vibration unit: influence of drive dynamics. Cybernetics and Physics, 8(4), pp. 298-306.

Tomchina, O. (2020). Control of oscillations in tworotor cyberphysical vibration units with time-varying observer. Cybernetics and Physics, 9 (4), pp. 206-213. Tomchina, O. P., Fradkov, A. L., Andrievsky, B. R., and Boikov, V. I. (2019). Angular velocity and phase 


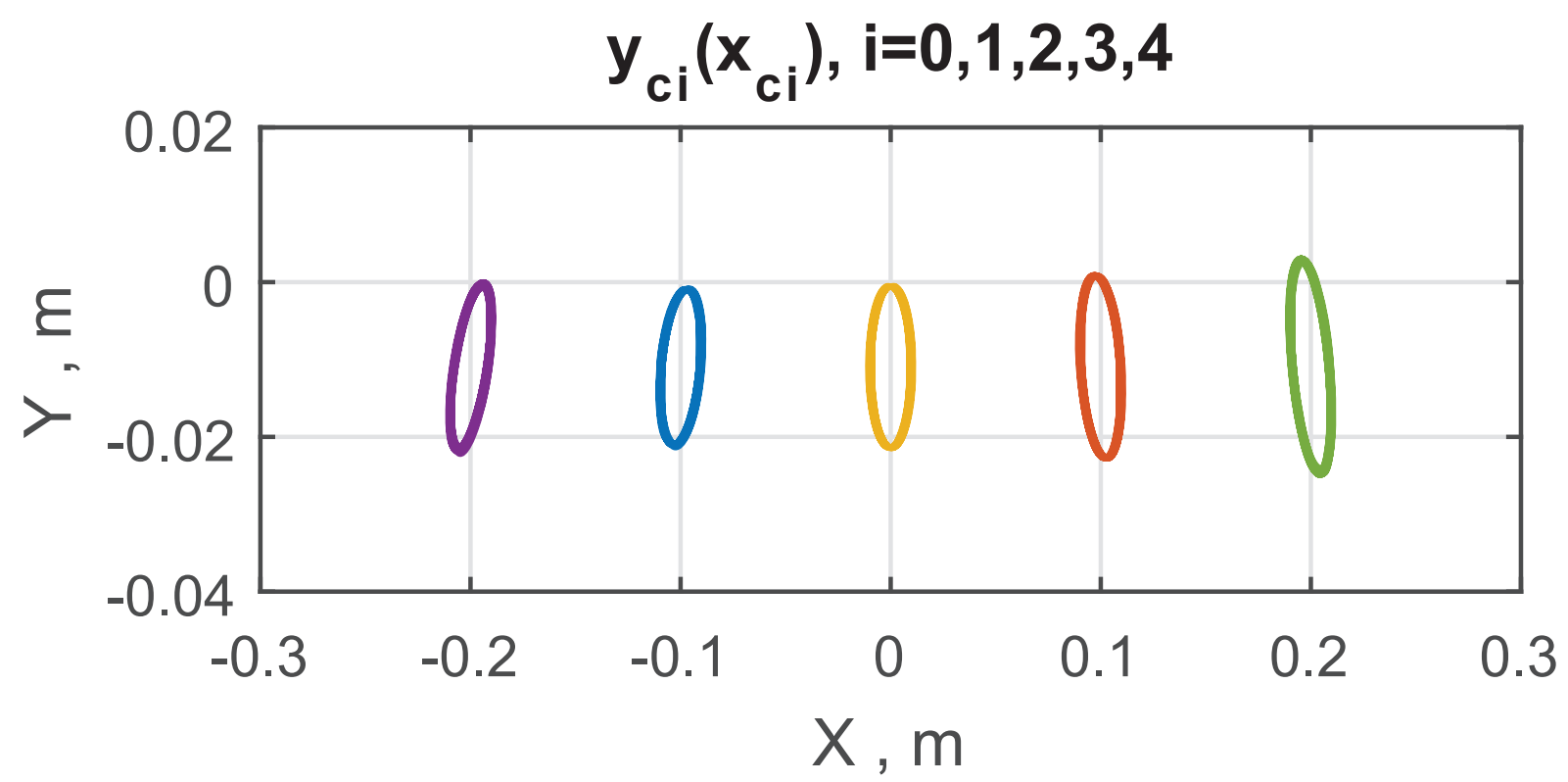

Figure 10. The shape of the VU vibration field at $c_{B}=500 \mathrm{Nm} / \mathrm{rad}, b_{B}=0.5 \mathrm{~N} \cdot \mathrm{m} \cdot \mathrm{s}, \Delta \varphi_{1}^{*}=-3.7 \mathrm{rad} ; \Delta \varphi=0.56 \mathrm{rad}$.

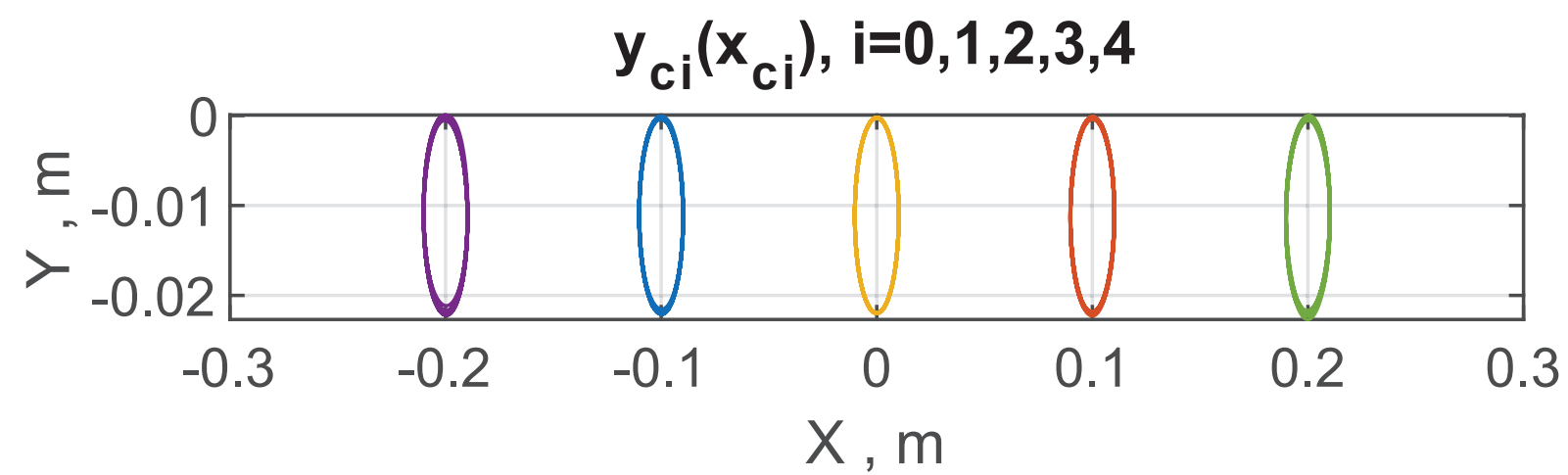

Figure 11. The type of the VU vibration field at $c_{B}=500 \mathrm{Nm} / \mathrm{rad}, b_{B}=0.5 \mathrm{~N} \cdot \mathrm{m} \cdot \mathrm{s}, \Delta \varphi_{1}^{*}=0 \mathrm{rad} ; \Delta \varphi=0.016 \mathrm{rad}$.

shift control of mechatronic vibrational setup. IFAC- PapersOnLine, 52 (15), pp. 436-441. 


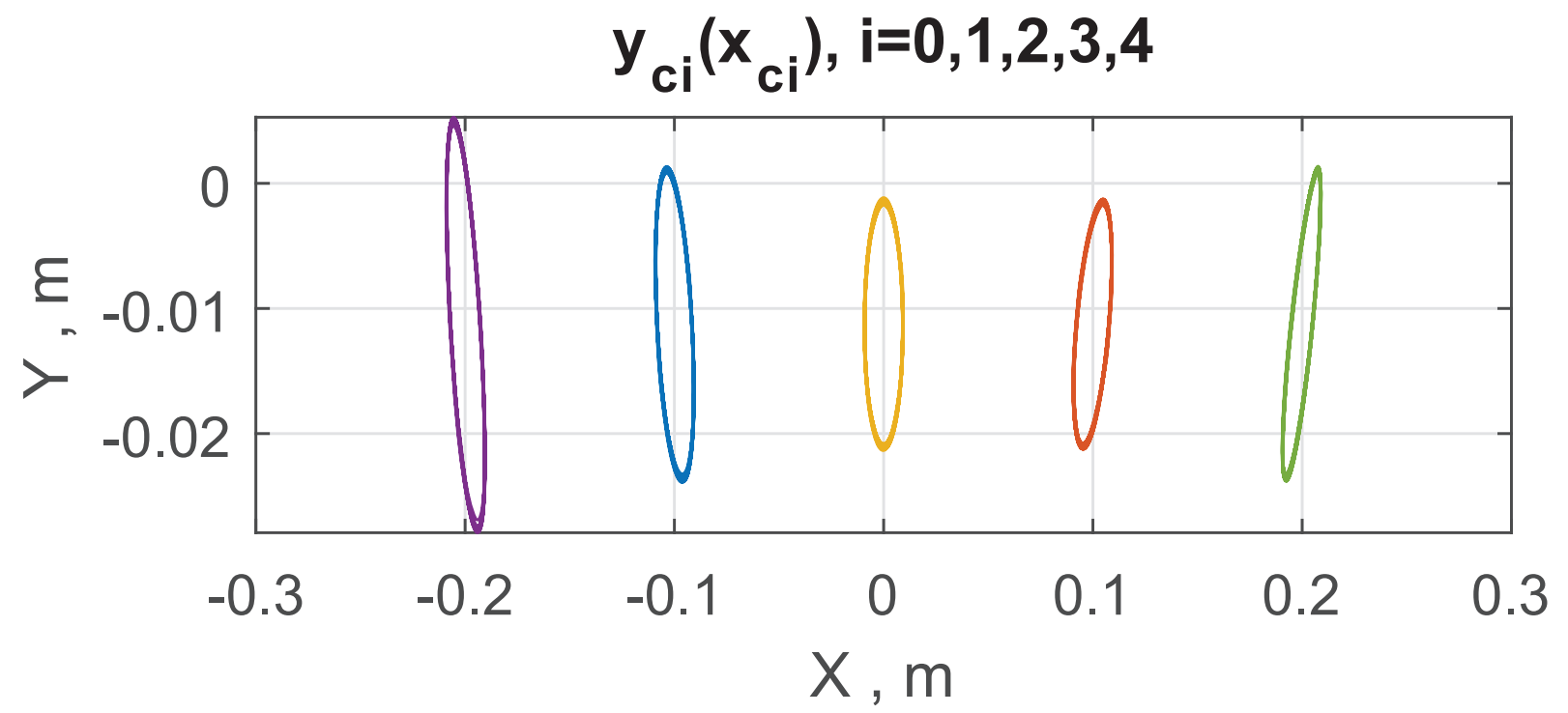

Figure 12. The type of the VU vibration field at $c_{B}=5000 \mathrm{Nm} / \mathrm{rad}, b_{B}=0.6 \mathrm{~N} \cdot \mathrm{m} \cdot \mathrm{s}, \Delta \varphi_{1}^{*}=5.7 \mathrm{rad} ; \Delta \varphi=-5.4 \mathrm{rad}$. 